Injections of whole pituitary gave varied results, and further work on this is being done.

These isolated results can contribute nothing conclusive to our knowledge as to the cause of eclipse plumage. Further experiments are in progress on the relation of the other endocrines to eclipse, mating, and female plumage. When these further experiments have been carried out and the full analysis is completed, it is hoped that interesting results on the subject of the endocrine modifications of plumage expression within the range of genetic determination will be obtained. Violet K. TALLENT.

National Institute of Poultry Husbandry, Newport, Salop.

1 Biol. Bull., 1910.

Proc. Zool. Soc. Lond., 1914

Arch. für Geflüg., 1930.

Proc. Roy. Soc. Edin., 1929.

\section{Change of Density of Carbon Disulphide with Temperature.}

THe investigations of Isnardi ${ }^{1}$ have shown that the value of the dielectric constant of carbon disulphide falls suddenly at $-90^{\circ}$. This drop appears at a considerable distance from the freezing point $\left(-112^{\circ}\right)$, so the phenomenon shows an analogy to the change of the dielectric constant of ethyl ether with temperature. On the basis of this analogy, it might be

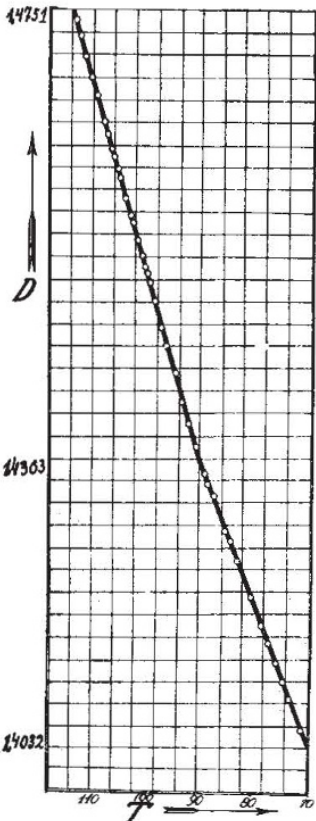

FIG. 1. reasonably supposed that the values of other physical properties of carbon disulphide would show similar abrupt if less marked changes at $-90^{\circ}$.

Experimental investigation has shown that on the heating curve of carbon disulphide there appears at $-90^{\circ}$ a distinct slowing down of rate of change of temperature. This suggests a change of the substance at this point from one liquid modification into another liquid modification. The analysis of the heating curve has enabled us to evaluate the latent heat for this change. ${ }^{2}$

The next step consisted in a study of the changes of density of carbon disulphide with temperature, with special attention to the neighbourhood of $-90^{\circ}$. I have therefore measured the density of carbon disulphide, using the method described by Kamerlingh Onnes and I. D. Boks. The details of the experiment have been described by me in the communications concerning the densities of ethyl ether and nitrobenzene. ${ }^{3}$

The temperatures were determined with an error not greater than $0.003^{\circ}$. The position of the meniscus of liquid could be read with an error of about $0.2 \mathrm{~mm}$.; this corresponds to an uncertainty of $1.5 \mathrm{~mm}^{3}$ in the value of volume of the liquid, so that, taking into account the volume of the dilatometer bulb, the relative error in the value of volume was about $0 \cdot 1^{\circ}$ per cent. The mass of the substance was determined with an error of about $0.0001 \mathrm{gm}$.; it follows that the values of the density were certain up to the fourth or even fifth figure.

As a freezing liquid I have used a mixture of ethyl ether and ethyl alcohol, mixed in a certain proportion. This mixture remains clear with the lowering of temperature, and it does not impede the motion of the stirrer even at $-120^{\circ}$

I may mention that the values of temperature were determined by means of the platinum resistance thermometers placed in the Dewar vessel at different depths. The determinations of temperature were made only after it was certain that the temperature was stationary throughout the volume of the freezing liquid. When the temperature of the freezing liquid was lowered down to $-120^{\circ}$ C., further cooling was stopped, whereas the stirrer remained in motion. Under the influence of the heat of the surroundings, the temperature of the carbon disulphide in the Dewar vessel increased slowly at the rate of about $1^{\circ}$ per hour.

The carbon disulphide used in this investigation was very carefully purified, starting from the pure $\mathrm{Cl}_{2}$ " pro analysi " delivered by Schering-Kahlbaum. The accompanying curve (Fig. 1) shows the change of density of carbon disulphide in the interval of temperatures between $112^{\circ} \mathrm{C}$. and $70^{\circ} \mathrm{C}$. It appears that with the lowering of temperature the density of carbon disulphide increases from the value 1.2628 at $20^{\circ}$ up to 1.4363 at $90^{\circ}$. At this temperature there begins a more rapid increase of density with lowering of temperature. In the neighbourhood of the freezing point the density has the value $1 \cdot 4751$.

It appears, therefore, that at the point of change from one to the other liquid modification of carbon disulphide the density curve shows a distinct change of slope.

Physical Laboratory, Technical Institute, Warsaw, July 20

1 Zeit. für Phys., 9, 153; 1922.

M. Wolfke and J. Mazur, NATURE, 127, 926; 1931

J. Mazur, NATURE, 127, 270; 1931; and 127, 893; 1931.

\section{Deep-Focus Earthquakes.}

Mr. F. J. ScRASE ${ }^{1}$ has recently found confirmation of the great depth assigned to certain earthquakes by the late Prof. $H$. H. Turner by recognising, in the data of the International Seismological Summary, the two separate branches of $P R_{1}$ and $S R_{1}$ that are to be expected if the focus be deep, and by observing the substantial agreement of their travel-times with the expected times calculated on the basis of the Zoeppritz-Turner tables. It may be of value to note in this connexion that we may, by the use of these reflected pulses, arrive at what is certainly a minimum value of the depth of focus, and this independently of any previous travel-time tables and even of any error in the assumed time of origin. This would eliminate any objection that might possibly be made to the use of the Zoeppritz-Turner tables.

All that is needed is good curves, derived from the data of the earthquake being investigated, showing the arrival times for $P$ and for $p P$ (this latter being the notation suggested by Scrase for a $P$ wave reflected close to the epicentre). It will be seen from such curves that the interval $p P-P$ gradually increases with increasing epicentral distance, though the two curves soon become nearly parallel. At a distance of $180^{\circ}$, where the slope of the two curves becomes equal to zero, the interval would represent exactly twice the time required for a wave to travel from focus to epicentre. Curves extending to that distance are not generally obtainable. However, we may choose a point where the two curves are almost parallel,

$$
\text { No. 3233, VoL. 128] }
$$

